\title{
DE LA GRÁFICA A LA ECUACIÓN, LA ARTICULACIÓN DE LOS DOS REGISTROS
}

No. 1

\section{GRAPHS AND EQUATIONS, THE ARTICULATION OF THE TWO RECORDS}

Forma de citar: PRADA-NÚÑEZ R, HERNÁNDEZ-SUÁREZ C. A. De la gráfica a la ecuacion, la articulación de los dos registros. Eco.Mat. 2014; 5(1): 49-59.

Recibido:

Junio 16 de 2014

Aceptado:

Agosto 25 de 2014
${ }^{1}$ Licenciado en Matemáticas

y Computación Universidad

Francisco de Paula Santander,

Cúcuta - Colombia. Magister en Matemática mención Educación

Matemática Universidad

Nacional Experimental del

Tachita, Venezuela. Docente de la Universidad Francisco de Paula Santander - Departamento de Matemáticas y Estadística, Cúcuta-Colombia. Correo electrónico: raulprada@ufps.edu.co

${ }^{2}$ Licenciado en Matemáticas y Computación Universidad Francisco de Paula Santander, Cúcuta-Colombia. Magister en Enseñanza de las Ciencias Básicas mención Matemáticas Universidad Nacional

Experimental del Tachita, Venezuela. Docente de la Universidad Francisco de Paula

Santander - Departamento

de Pedagogía, Andragogía,

Comunicación y Multimedios. Cúcuta - Colombia. Correo electrónico: cesaraugusto@ufps.edu.co

\author{
Prada Núñez, $\mathrm{R}^{1}$ y Hernández Suárez, C. A. ${ }^{2}$
}

\section{RESUMEN}

Este artículo es el producto de un estudio exploratorio que considera como referencia una propuesta pedagógica que surgió como alternativa de solución a los problemas identificados por una estudio previo adelantado por un grupo de docentes que hacen investigación en Educación Matemáticas de la Universidad Francisco de Paula Santander (UFPS) y que propone desarrollar temas matemáticos a partir del registro gráfico e intentando articularlo con el registro algebraico, es decir, realizar el proceso inverso a la que tradicionalmente se realiza en el aula. Se toma como marco de referencia los trabajos de Duval y Hitt, basado en los registros de representación semiótica y su incidencia en el aprendizaje de conceptos matemáticos, en particular el concepto de función real. A partir de la aplicación de un cuestionario a un grupo de 109 estudiantes de la Facultad de Ingeniería se exponen algunos de los resultados sobre la articulación de la representación gráfica a la algebraica.

Palabras Clave: Representaciones semióticas, registro gráfico, articulación de registros.

\section{ABSTRACT}

This article is the product of an exploratory study that considers a pedagogical reference emerged as an alternative solution to the problems identified by a previous study conducted by a group of teachers who do research in Mathematics Education at the University Francisco de Paula Santander (UFPS) and aims to develop mathematical topics from graphic recording and trying to link it to the algebraic register, ie the reverse process which traditionally takes place in the classroom. It takes as a reference the work of Duval and Hitt, based on the records of semiotic representation and its impact on the learning of mathematical concepts, particularly the concept of real function. From the application of a questionnaire to a group of 109 students of the Faculty of Engineering, some results on the structure of the graphic representation to algebraic exposed.

Keywords: semiotic representations, graphic recording, articulation of records. 
No. 1

L. os resultados que aquí se presenta es parte de una investigación más amplia, tendiente a buscar alternativas de solución a dificultades detectados en la enseñanza de la Matemática en los primeros semestres de los programas académicos la Facultad de Ingeniería de la UFPS, Cúcuta Colombia.

El Cálculo es una de las primeras asignaturas básicas con que se encuentra el estudiante cuando ingresa a la Universidad en los programas de ingeniería, ya que es un pilar en su formación. En esta asignatura se brinda al estudiante los conocimientos básicos que le servirán de herramientas al llegar a las materias propias de su especialidad.

Una de las observaciones más frecuentes encontradas por los docentes de Cálculo al analizar las evaluaciones parciales o finales son los errores que cometen los estudiantes atribuibles a deficiencias de aprendizaje provenientes de los niveles educativos anteriores y no relacionadas directamente con los temáticas propias de las asignaturas de Matemáticas que cursan, lo que indica que los mismos comparten un marcado déficit en su formación Matemática dando resultados negativos.

Duval (1988) en su artículo "Gráficas y ecuaciones - La articulación de dos registros" aborda las reglas de correspondencia semiótica entre el registro de las representaciones gráficas y el de la escritura algebraica y argumenta que, fundamentalmente, la razón de las dificultades de lectura y de interpretación de las representaciones gráficas cartesianas es el desconocimiento de éstas. De acuerdo a Duval, las propiedades de las figuras generalmente no son abordadas en la enseñanza y al desconocer la especificidad y la importancia de esta vía, no puede alcanzarse el objetivo de una utilización correcta de las gráficas cartesianas por la mayoría de los estudiantes.

Diversas investigaciones han detectado importantes dificultades en los estudiantes en el campo conceptual de la Matemática. Hitt
(1998), asegura que los estudiantes de Ingeniería, después de llevar un curso de Cálculo, no logran resolver problemas no rutinarios; sugiere que los métodos tradicionales de enseñanza del cálculo son insuficientes en la preparación de buenos estudiantes para aplicar el Cálculo de manera creativa. En los programas de Ingeniería, se exige una comprensión profunda de los conceptos de cálculo, en particular del concepto de "función", el cual es fundamental para el desarrollo de esta asignatura y su objetivo principal en su enseñanza es que los estudiantes sean capaces de distinguir entre sus diferentes usos y los puedan aplicar en materias de su especialidad.

No obstante, su aprendizaje representa grandes dificultades a los estudiantes. Hitt (1996) expresa que "la aprehensión del concepto de función no parece una tarea fácil, la gran cantidad de investigaciones realizadas con estudiantes para detectar obstáculos en el aprendizaje del concepto confirman la dificultad de aprensión del concepto"... luego agrega "tienen una fuerte tendencia en pensar en funciones continuas y, en muchos casos les falta habilidad para construirlas"...

Referido a ello, uno de los aspectos a ser considerado es la posible ausencia de articulación entre distintos registros de representación de los conceptos. Para determinar condiciones previas en ese sentido, se realizó una prueba o cuestionario, pensando en que los problemas de coordinación entre las representaciones grafica a la algebraica de los objetos matemáticos podían verse reflejado en los resultados de la misma.

\section{MARCO TEORICO}

La mayoría de las investigaciones en Educación Matemática en el ámbito universitario han sido realizadas sobre los conceptos del Cálculo, sin embargo, en muy pocas se ha hecho investigación directa sobre la manera de cómo se imparten los conceptos. La mayoría de estos estudios tratan de encontrar obstáculos que presentan los estudiantes cuando aprenden los conceptos de este tema; muchos investigadores lo 
enmarcan exclusivamente como un problema de aprendizaje, dejándole al estudiante toda la responsabilidad de estos obstáculos; sin embargo, ¿Tienen los profesores alguna responsabilidad sobre lo que aprenden sus estudiantes?, es decir, ¿los profesores saben cómo abordar el tema de la mejor forma de modo que se pueda garantizar que sus estudiantes de apropien del concepto?

Desde el punto de vista de varios investigadores en el tema, los estudiantes muestran obstáculos cognitivos porque no han construido el concepto matemático en cuestión y ellos han desarrollado concepciones de ese concepto, que los lleva a situaciones de éxito en ciertos contextos y de fracaso en otros. Es decir, una concepción es un conocimiento que se tiene sobre un concepto particular, este conocimiento tiene un campo de validez y se va refinando con la experiencia; en otras palabras, la concepción se formula a partir de ejemplos particulares y en un inicio se utiliza una definición intuitiva del concepto con el uso de palabras sencillas; con el paso del tiempo esta nueva definición se va refinando hasta que llega (en el mejor de los casos) a tomar en cuenta el caso general.

Las concepciones pueden ser un obstáculo en el proceso de enseñanza o en la resolución de problemas cuando están poco refinadas ya que no permiten identificar o construir correctamente ejemplos del concepto con el que se está trabajando. Sin embargo, el desarrollo de concepciones es el paso natural para la construcción de conceptos matemáticos, sobre todo si esos conceptos son de carácter complejo.

Una pregunta a responder es ¿qué factores intervienen en los procesos de enseñanza que mejoran el aprendizaje de los estudiantes? ante esta pregunta la lista se hace interminable: algunos investigadores proponen como factor de incidencia la intensidad semanal de clase a que tienen acceso los estudiantes, otros proponen el uso de TIC, entre otras. "La enseñanza tradicional tiende a centrarse en una práctica algorítmica y algebraica", según Artigue (1995). Santos y Vargas (2003) señalan que "el tipo de actividades que se fomenten en el salón de clase necesariamente influyen en el tipo de aprovechamiento que los estudiantes exhiban en sus procesos de entendimiento o resolución de problemas".

Lo anterior ubican el proceso de enseñanza que se practica en el aula como uno de los factores de mayor incidencia en la aprehensión de conceptos matemáticos, pero ¿es fácil la aprehensión de conceptos matemáticos? Para dar respuesta a esta pregunta, vale la pena citar lo afirmado por Duval desde el punto de vista de su enfoque teórico, un concepto se va construyendo mediante tareas que implique el uso de diferentes sistemas dc representación y promuevan la articulación coherente entre sus representaciones. O lo afirmado por Hitt, el conocimiento de un individuo sobre un concepto es estable si es capaz de articular diferentes representaciones de ésta, libre de contradicciones (2001).

En panicular, Duval (1988) sostiene que con lo anteriormente expuesto queda clara la existencia de dificultades en el proceso de enseñanza - aprendizaje de los estudiantes de distintos niveles de escolaridad alrededor de los diversos conceptos matemáticos, ello como posible efecto de la aplicación de un sistema tradicional de enseñanza que ha favorecido la utilización de unos registros más que otros.

Duval (1995) distingue entre lo que llama objetos matemáticos y sus representaciones, y sostiene que estas últimas tienen un papel indispensable en la aprehensión del objeto o concepto matemático. Este autor establece que: "toda representación es parcialmente cognitiva con respecto a lo que representa" y por tanto: "la comprensión (integral) de un contenido conceptual está basada en la coordinación de, al menos, dos registros de representación, en nuestro caso el registro gráfico y el simbólico en funciones afines, $y$ esta coordinación queda de manifiesto por medio del uso rápido y la espontaneidad de la conversión (la transformación de un objeto, de una situación o de una información dada en un registro, en una representación
Enero-Diciembre 2014 ISSN 1794-8231 PP: 49-59 
No. 1

Enero-Diciembre 2014 ISSN 1794-8231

PP: 49-59 de este mismo objeto, esta misma situación $o$ de la misma información en otro registro) cognitiva".

Propuesta pedagógica. El objetivo de esta propuesta es incorporar en el proceso de enseñanza del aula los diversos registros de representación semiótica y su respectiva articulación con el fin de garantizar en el estudiante mayor cantidad de argumentos matemáticos al momento de resolver ejercicio o problemas. La propuesta aborda diversos conceptos del Cálculo diferencial tomando como punto de partida en cada caso el uso del registro grá- fico y a partir de él desarrollar un completo análisis matemático de los planteamientos, tales como calcular dominio y rango, continuidad, derivadas e inversas de funciones, entre otros aspectos.

Funciones básicas. Al empezar el estudio de las Matemáticas a partir del registro de representación gráfico, se debe conocer la existencia de una serie de funciones básicas que en algunos casos sufren algunas transformaciones y de esa forma dan lugar a un innumerable número de funciones adicionales (Tabla 1).

Tabla 1. Funciones básicas

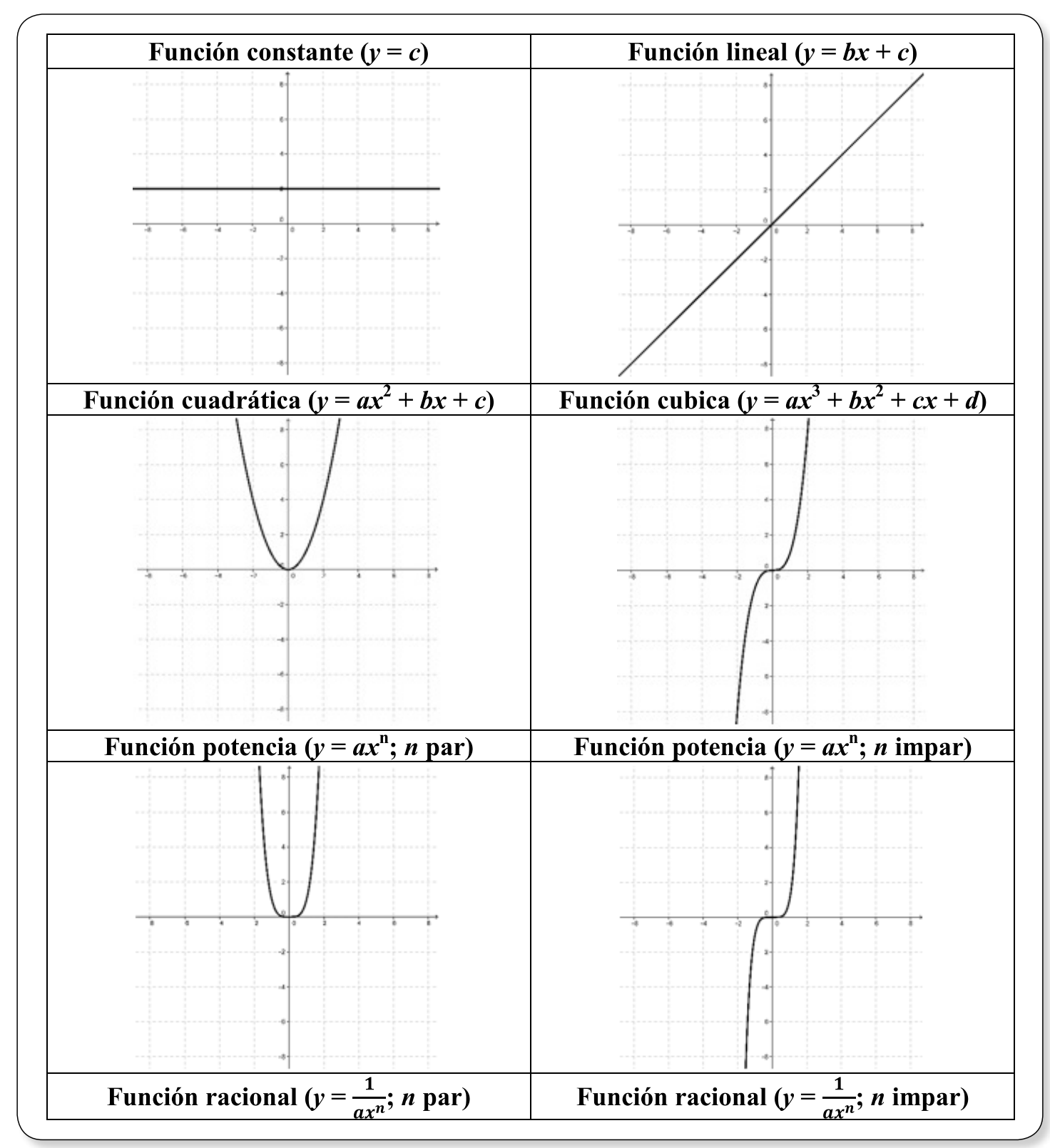


Enero-Diciembre 2014 ISSN 1794-8231

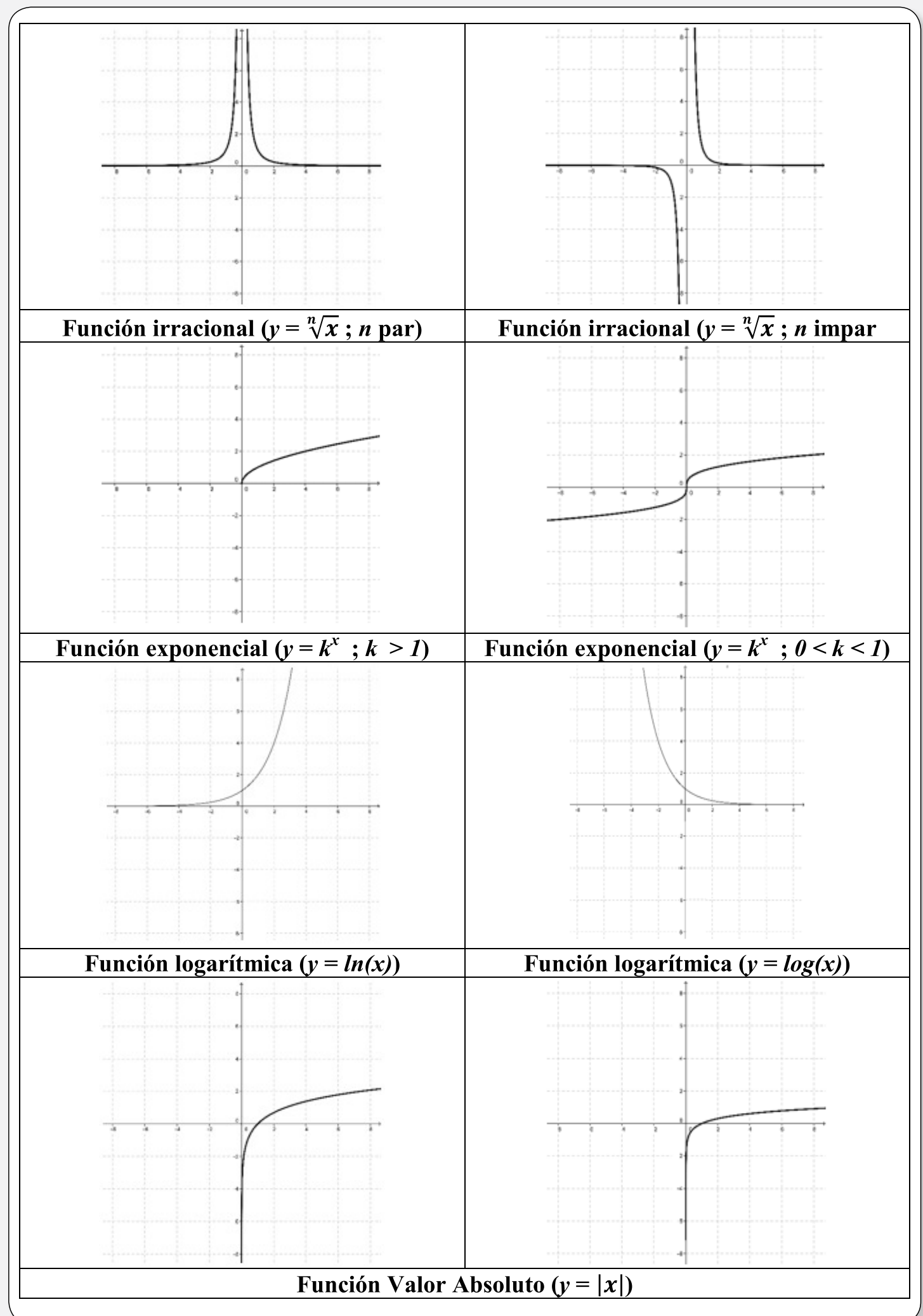




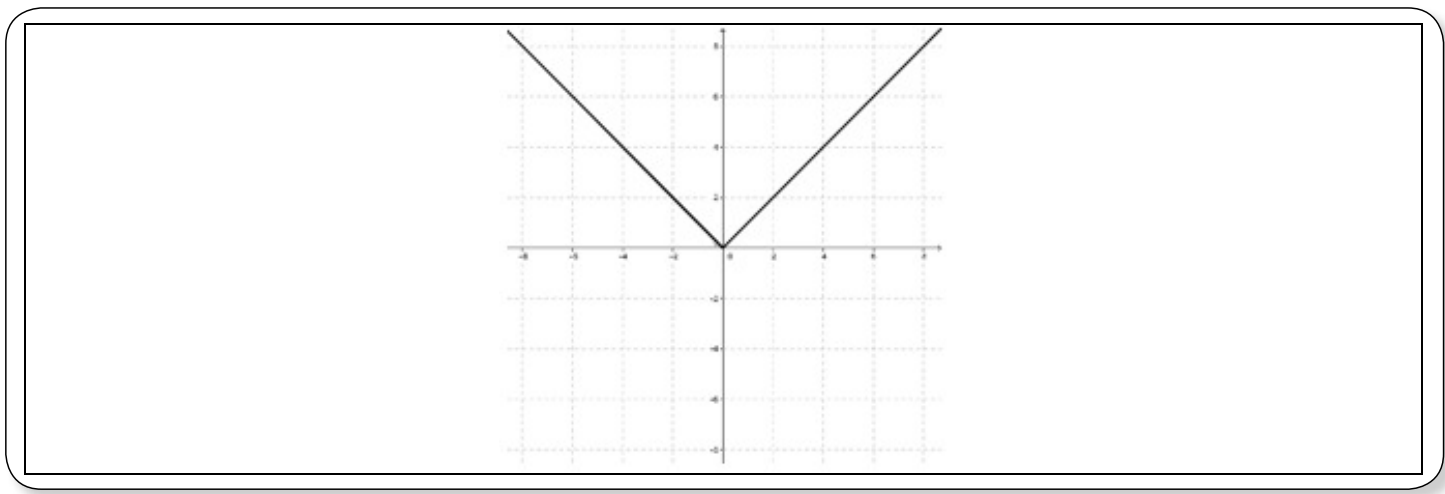

Principios básicos de la graficación. Existen cuatro principios básicos de la graficación los cuales se relacionan a continuación:

Principio de alargamiento y reducción (La gráfica de $f$ se alarga verticalmente en un factor $c$ o comprime verticalmente en un factor $1 / c)$.

Este principio también se llama ampliación o reducción. Otro nombre que recibe es el de elongación o compresión.
Principio de desplazamiento vertical (La gráfica de $f$ se desplaza verticalmente hacia arriba o abajo una distancia $c$ ).

Principio de desplazamiento horizontal (La gráfica de $f$ se desplaza horizontalmente a la derecha o izquierda una distancia $c$ ).

Los principios de desplazamiento también se les llaman traslaciones o corrimientos.

Principio de reflexión.

Tabla 2. Principios básicos de graficación sobre la función $f(x)=x^{2}$

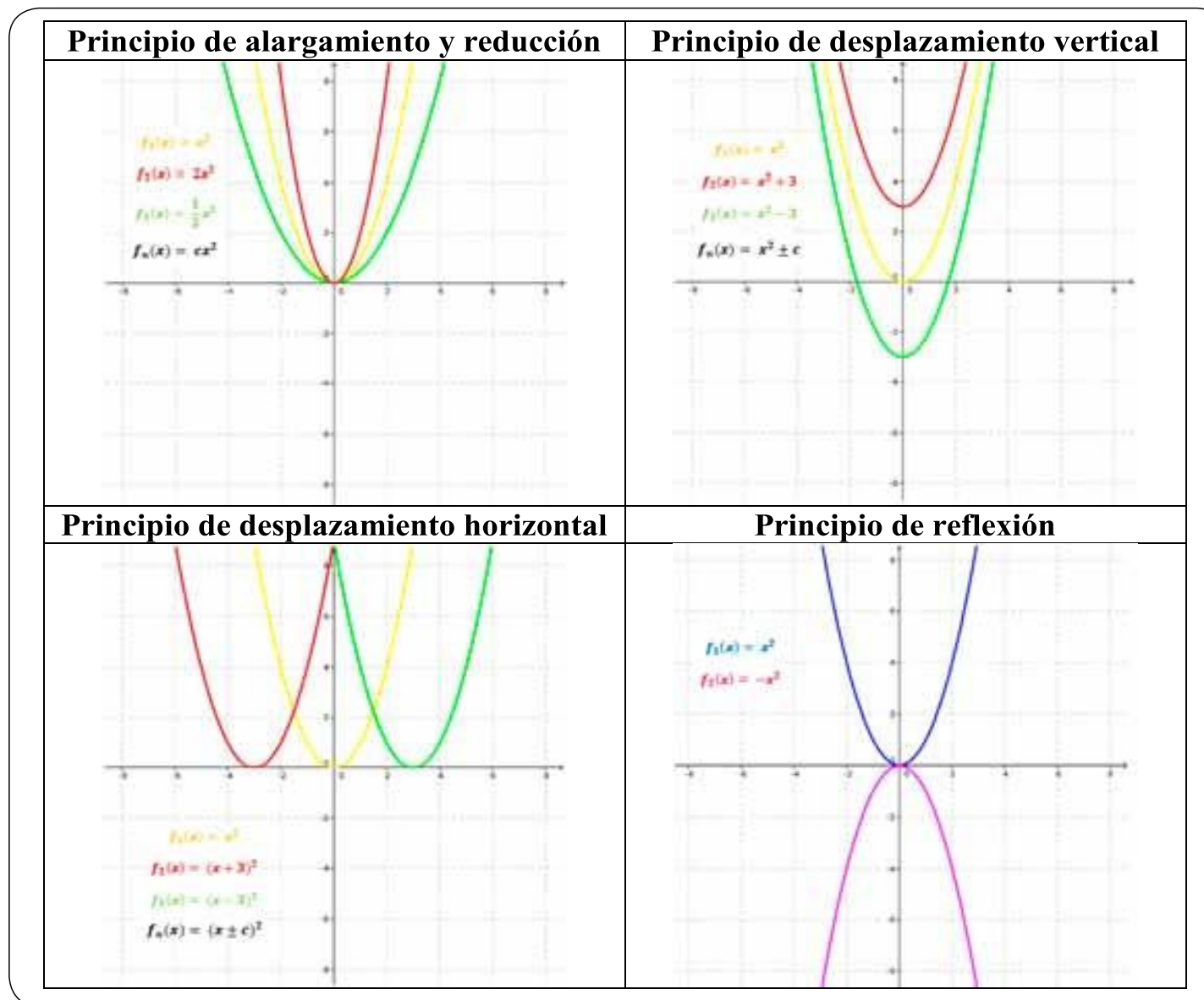




\section{MATERIALES Y METODO}

El estudio respondió a un enfoque exploratorio y descriptivo. Con el fin de obtener elementos de juicio sobre los posibles logrosy dificultades de los estudiantes en la articulación de los sistemas gráfico al algebraico, se aplicó una prueba o cuestionario que se administró a 109 estudiantes, de entre 17 y 20 años, de los dos primeros semestres de la Facultad de Ingeniería de la UFPS.

El instrumento a utilizar fue sometido a diversas instancias de evaluación, tanto por integrantes del equipo de investigación como por especialistas en educación Matemática. El resultado de las reestructuraciones y reformulaciones de diseño fue la versión final del cuestionario aplicado a los estudiantes. El cuestionario está compuesto de cinco situaciones problema relacionadas con el concepto de función real.

\section{RESULTADOS Y DISCUSIÓN}

Articulación de los registros grafico al algebraico. Con el fin de dar el primer paso en la investigación de las dificultades que presentan los estudiantes cuando el docente rompe el esquema de estudio tradicional de la enseñanza de las funciones reales, es decir, se considera como información inicial la representación gráfica de una función $f$ $(x)$ y a partir de ella se proponen una serie de cuestionamientos en los que para dar solución a cada uno de ellos el estudiante debe interpretar la información que contiene el gráfico, debe aplicar los principios mencionados anteriormente y debe razonar sobre la veracidad de algunas hipótesis. Se propuso un instrumento básico compuesto de 4 ítems y se le aplicó a un grupo de 109 estudiantes de los dos primeros semestre de los diversos programas de la Facultad de Ingenierías de la UFPS.

Los ejercicios propuestos y sus resultados se analizan a continuación:
Ejercicio 1. Dada la siguiente gráfica de la función $f(x)$.

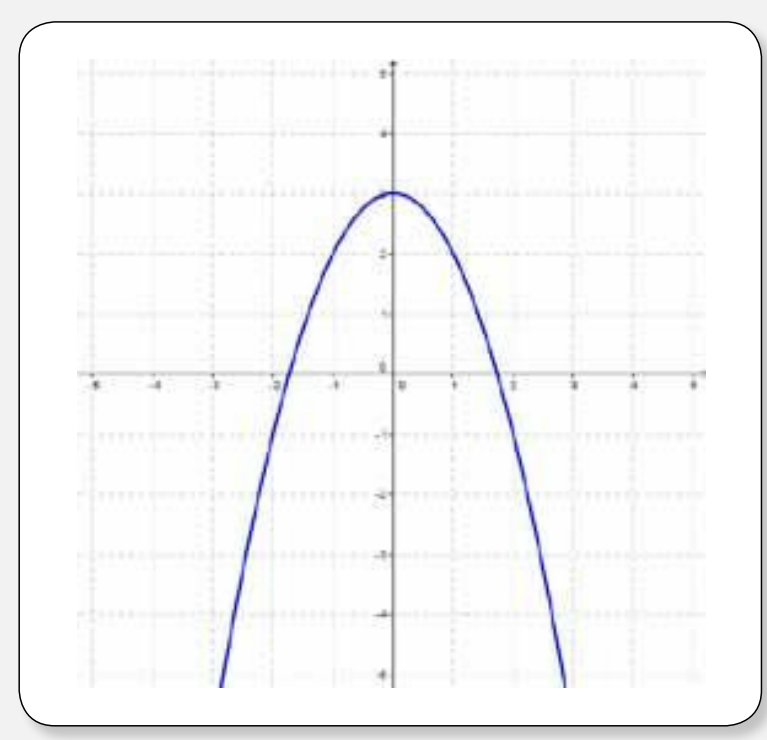

Determine:

Determine:
a. Qué tipo de función es?

El $50 \%$ de los estudiantes encuestados afirmaron que la gráfica corresponde a una función cuadrática, un 30\% mencionan una función diferente y el porcentaje restante no responde la pregunta.

\section{b. Halle su ecuación}

El $40 \%$ de los estudiantes encuestados proponen la estructura de la ecuación correcta, un $20 \%$ proponen cualquier tipo de ecuación y el porcentaje restante no realiza proceso alguno.

c. Determine el dominio y el rango

El $50 \%$ de los estudiantes encuestados determinan el dominio y el rango de la función de forma correcta utilizando la notación de intervalos diferenciando entre intervalos abiertos y cerrados; el 20\% identifican sólo el rango y el porcentaje restante no propone proceso alguno.

d. ¿La función es continua?

El $50 \%$ de los es estudiante encuestados afirman que la información es continua en todo 
No. 1

Enero-Diciembre 2014 ISSN 1794-8231

PP: 49-59 su recorrido pero no proponen argumento alguno; un 10\% afirma la continuidad de la función en un solo punto y el porcentaje restante no responde.

e. Halle los intervalos dónde la función es creciente y dónde es decreciente

Sólo el 10\% de los estudiantes encuestados proponen de forma adecuada los intervalos en dónde la función es decreciente y en los que es creciente, el porcentaje restante no respondió a éste item.

f. Determine gráfica y analíticamente su derivada

Sólo el 10\% de los estudiantes encuestados determinaron la derivada de la función tanto de forma gráfica como analítica y el resto de los estudiantes no desarrollaron proceso alguno en éste item, evidenciando dificultad en el tema.

Ejercicio 2. Dada la gráfica de la función $f(x)$.

a. Halle la expresión algebraica asociada a la gráfica de $f(x)$.

El $50 \%$ de los estudiantes encuestados propusieron la ecuación de la gráfica de forma correcta y el porcentaje restante no realizó proceso alguno.

b. Grafique la función $g(x)=|f(x)|$

La totalidad de estudiantes encuestados presentaron dificultad ya que el $90 \%$ de ellos no realizaron proceso alguno y el 10\% restante determinaron dominio y rango pero la formulación de la ecuación es incorrecta.

Ejercicio 3. Dada la gráfica la función $\mathrm{f}(x)$.

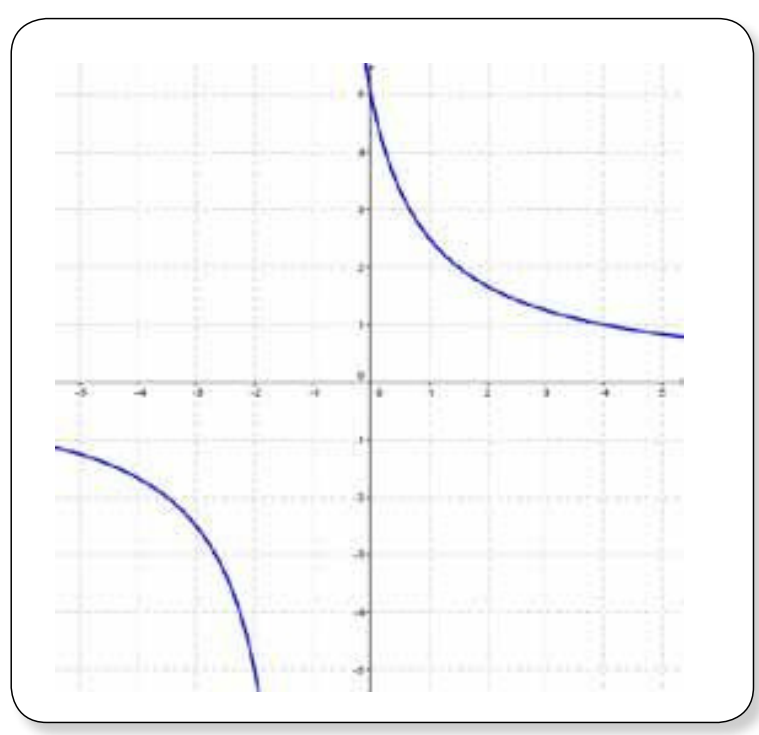

a. Halle la expresión algebraica asociada a la gráfica de

El $10 \%$ de los estudiantes encuestados asocian la gráfica con una función racional pero la expresión propuesta está errada ya que no considera en la expresión el intercepto con el eje " $Y$ ", un $30 \%$ construyen tablas de valores obtenidos de la gráfica pero no proponen expresión alguna y el porcentaje restante no responden.

b. Grafique la función

El 100\% de los estudiantes encuestados presentaron dificultad en este aspecto, al punto que ninguno de ellos intentó realizar proceso alguno. 
Ejercicio 4. Dada la gráfica de la función $f(x)$.

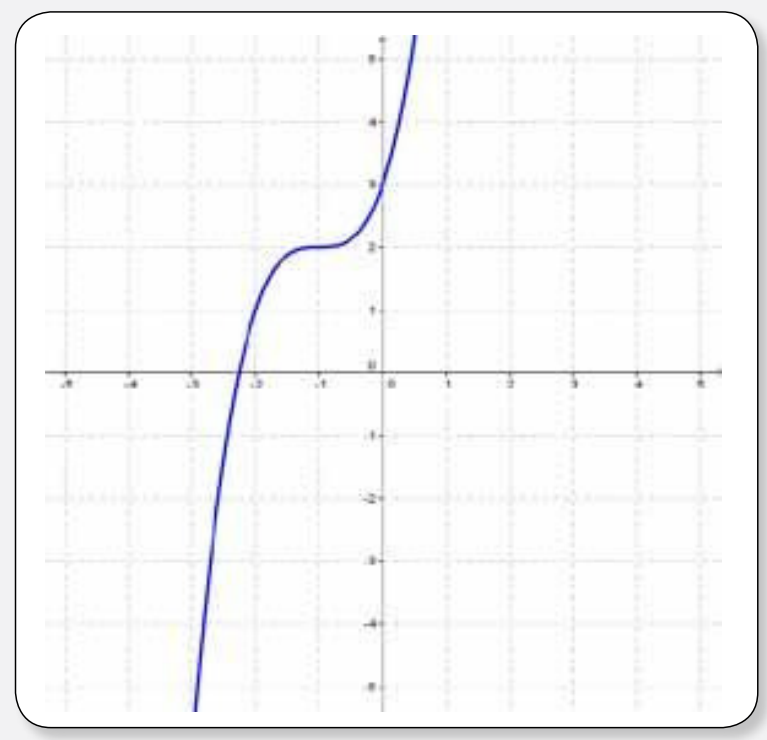

a. Halle la expresión algebraica asociada a la gráfica de $f(x)$.

El $80 \%$ de los estudiantes encuestados no realizaron proceso alguno, sólo el 10\% de ellos intentaron proponer una expresión algebraica considerando los principios de desplazamiento vertical y horizontal conjuntamente pero presentaron dificultad en la ubicación del exponente de la expresión y el 10\% restante asocian la gráfica con una función potencia con exponente impar pero las expresiones propuestas son incorrectas.

b. Determine la ecuación de la función inversa de $f(x)$, es decir, $f^{-1}(x)$.

La totalidad de estudiantes encuestados no propusieron proceso alguno. Vale la pena destacar que del 10\% de estudiantes que habian intentado construir una expresión algebraica considerando los principios de desplazamiento horizontal y vertical no mostraron ni el más mínimo interés en la búsqueda de la función inversa.

c. Grafique $f^{-1}(x)$ y determine sus características.

Ninguno de los estudiantes realizó proceso alguno.

\section{CONCLUSIONES}

Como se puede observar el instrumento propuesto inicia con el estudio de las características asociadas a la función cuadrática apoyándose en los conceptos básicos que son abordados en los dos grados de la media vocacional tal como lo referencian los estándares curriculares en Matemáticas. Se consideran de cada una de ellas los conceptos de clasificación de funciones, construcción de la expresión algebraica asociada a la gráfica, dominio, rango, continuidad de una función, intervalos de crecimiento y decrecimiento así como su derivada.

De los resultados obtenidos se encontraron dificultades en un alto porcentaje de estudiantes al proponer la expresión algebraica asociada con la gráfica representada, situación que contrasta con lo afirmado anteriormente ya que el proceso de formación tradicional de los docentes es partir de la expresión algebraica de la función hasta representarla gráficamente en el plano cartesiano pasando por el registro tabular como registro intermedio.

Una vez el estudiante tiene la representación gráfica, se apoya en ella para identificar el dominio y el rango de la función, situación que se evidenció en la muestra. Alrededor del concepto de continuidad, los estudiantes empiezan a exhibir métodos empíricos de validación que se alejan de la rigurosidad Matemática y en el momento que deben justificar su proceder mientras les funcione lo justifican pero en caso de encontrar inconsistencias, de inmediato evitan la confrontación omitiendo la argumentación en la respuesta.

En los conceptos de función creciente y función decreciente el estudiante evita proponer respuesta alguna a pesar de tener la representación gráfica de la función en la que se podía apoyar, lo que deja de manifiesto la existencia de vacíos conceptuales en lo que respecta a la relación entre las variables e y su representación en el plano cartesiano, que sumado a la dependencia que se tiene de recurrir al registro algebraico para validar las relaciones entre las variables terminan convirtiéndose en 
No. 1

Enero-Diciembre 2014 ISSN 1794-8231

PP: 49-59 un gran obstáculo conceptual en el estudiante; con respecto al proceso de derivación la situación no mejora, un pequeño porcentaje de estudiantes asocia la derivada de la función con una función lineal cuya pendiente es negativa debido a que la concavidad de la función cuadrática está orientada hacia el eje negativo de las Y.

En la segunda situación propuesta se aborda el concepto de la función valor absoluto y lo que se buscaba era identificar si el estudiante podía representar de forma gráfica esta función sin utilizar el registro algebraico, apoyándose solamente en el hecho de saber que la función valor absoluto arroja siempre valores positivos para la variable dependiente, luego con esta información y apoyándose en el principio de reflexión poder graficar la función solicitada.

Como se pudo observar en la muestra los estudiantes no realizaron proceso alguno evidenciando el posible desconocimiento alrededor del comportamiento de esta función ya que en muchas instituciones educativas el docente pierde sesiones de clases debido al cruce con actividades institucionales llevándolo a tener que seleccionar algunas temas para dejarlos fuera del desarrollo de la asignatura y la función valor absoluto es excluida comúnmente.

En la tercera situación visualiza una función racional en dónde se evidencia la presencia de asíntotas tanto horizontales como verticales que afectan la continuidad de la función, así como el concepto de intercepto con el eje Y. El identificar desde la gráfica estos elementos característicos de la función racional proporciona elementos valiosos para la construcción de la expresión algebraica asociada a la gráfica. Pero dicha situación demanda del estudiante claridad conceptual alrededor del tema y la aprehensión de estos conceptos, situación que se dificulta debido al proceso tradicional de enseñanza que proponen comúnmente los docentes del área. Los resultados obtenidos de la muestra se convierten en evidencia para confirmar la dificultad que presentan los estudiantes alrededor del tema de funciones reales.
En la cuarta situación planteada aborda los conceptos de función potencia con exponente impar, así como los principios de desplazamiento horizontal y vertical afectan la representación gráfica de la función para finalizar con el concepto de función inversa. Como se evidenció en la muestra a medida que las situaciones propuestas exigían de los estudiantes mayor nivel de conocimiento de las funciones reales y de los principios básicos de graficación, el nivel de no respuesta fue creciendo al punto de alcanzar el $100 \%$ en la última situación planteada.

Los resultados encontrados abren la puerta a seguir investigando en el tema con el fin de mejorar el proceso de caracterización de las dificultades que presentan los estudiantes cuando se tiene el registro gráfico como punto de partida en la obtención de diversos elementos constitutivos y característicos de las diversas funciones reales. Todo ello con el fin de sumar argumentos que sirvan de motivación a los docentes de Matemáticas a transformar sus prácticas en dónde se propicie la correcta articulación de los diversos tipos de representaciones semióticas.

\section{REFERENCIAS BIBLIOGRÁFICAS}

Artigue. M. (1995). La enseñanza de los principios del cálculo: problemas epistemológicos, Cognitivos y didácticos. P. Gómez (Ed), Ingeniería didáctica en Educación Matemática. Un esquema para la investigación y la Innovación en la enseñanzaaprendizaje de las Matemáticas. (p. 97-140). México, Grupo editorial Iberoamérica.

Duval R. (1988) Graphiques et equations: l'Articulation de deux registres. Anales de Didactique et de Sciences Cognitives. Traducción: Gráficas y ecuaciones: la articulación de dos registros. En Antología en Educación Matemática (Editor E. Sánchez). Departamento de Matemática Educativa del Cinvestav-IPN, México.

Duval R. (1995) Sémiosis et pensée humaine: 
Registres sémiotiques et apprentissage

Enero-Diciembre 2014 intellectuels. Peter Lang, Suisse.

Hitt, F. (1996). Sistemas semióticos de representación del concepto de función y su relación con problemas epistemológicos y didácticos. En F. Hitt (Ed.) Investigaciones en Matemática Educativa. (pp.245-264). México: Grupo Editorial Iberoamérica.

Hitt, F. (1998). Visualización matemática, representaciones, nuevas tecnologías y curriculum. Educación Matemática, 10 (2), 23-45.

Hitt, F. (2001). El papel de los esquemas, las conexiones y las representaciones internas y externas dentro de un proyecto de investigación en Educación Matemática. En Gómez, P., y Rico, L. (Eds.). Iniciación a la investigación en didáctica de la Matemática. Homenaje al profesor Mauricio Castro. Granada: Editorial Universidad de Granada.

Santos, M. \& Vargas, C. (2003). Más allá del uso de exámenes estandarizados. Avance y Perspectiva, 22, pp. 9-22. 